\title{
Damped Newton's Method on Riemannian Manifolds
}

\author{
Marcio Antônio de A. Bortolotia, Teles A. Fernandes ${ }^{\mathrm{a}, *}$, Orizon Pereira \\ Ferreira $^{\mathrm{b}}$, Yuan Jin Yun ${ }^{\mathrm{c}}$ \\ ${ }^{a}$ DCET/UESB, CP-95, CEP 45083-900-Vitória da Conquista, Bahia, Brazil \\ ${ }^{b} I M E / U F G, C P-131, C E P$ 74001-970 - Goaiania, Goiás, Brazil \\ ${ }^{c} D M / C P / U F P R$, Jardin das Américas, CEP 81531-980 - Curitiba, Paraná, Brazil
}

\begin{abstract}
A damped Newton's method to find a singularity of a vector field in Riemannian setting is presented with global convergence study. It is ensured that the sequence generated by the proposed method reduces to a sequence generated by the Riemannian version of the classical Newton's method after a finite number of iterations, consequently its convergence rate is superlinear/quadratic. Moreover, numerical experiments illustrate that the damped Newton's method has better performance than the Newton's method in number of iteration and computational time.
\end{abstract}

Keywords: Global Optimization, Damped Newton method, Superlinear/Quadratic Rate, Riemannian Manifold

AMS subject classifications: 90C30, 49M15, 65K05.

\section{Introduction}

In the 1990s, the optimization on manifolds area gained considerable popularity, especially with the work of Edelman et al. [1. Recent years have witnessed a growing interest in the development of numerical algorithms for nonlinear manifolds, as there are many numerical problems posed in manifolds arising in various natural contexts, see [1, 2, 3]. Thus, algorithms using the

\footnotetext{
* Corresponding author

Email addresses: mbortoloti@uedb.edu.br (Marcio Antônio de A. Bortoloti), telesfernades@uesb.edu.br (Teles A. Fernandes), orizon@ufg.br (Orizon Pereira Ferreira), jin@ufpr.br (Yuan Jin Yun), support@elsevier.com (Yuan Jin Yun)
}

Preprint submitted to Journal of ${ }^{A} T_{E} X$ Templates

July 20, 2018 
differential structure of nonlinear manifolds play an important role in optimization; see [4, 5, 6, 7, 8, 9, 10, 11, 12, 13, 14, 15. In this paper, instead of focusing on finding singularities of gradient vector fields, including local minimizers, on Riemannian manifolds, we consider the more general problem of finding singularities of vector fields.

Among the methods to find a zero of a nonlinear function, Newton's method under suitable conditions has local quadratic/superlinear convergence rate; see [16, 17]. This remarkable property has motivated several studies on generalizing Newton's method from a linear setting to the Riemannian one [3, 18, 19, 20, 21, 22, 23, 24. Although of Newton's method shows fast local convergence, it is very sensitive with respect to the initial iterate and may diverge if it is not sufficiently close to the solution. To overcome this drawback, some strategies used in Newton's method for optimization problems were introduced including BFGS, Levenberg-Marquardt and trust region etc, see [16] and 25. On the other hand, it is well-known in the linear context that one way to globalize the convergence of Newton's method is to damp its step-size (see [16, 26, 25, 27]). Among the strategies used, one particularly interesting is the one by using a linear search together with a merit function. In this case, the basic idea is to use linear search to damp Newton step-size when the full step does not provide a sufficient decrease for values of the chosen merit function, which measures the quality of approximation to a zero of the nonlinear function in consideration. Newton's method with these globalization strategies are called Damped Newton's Methods. For a comprehensive study about these subject on linear setting see, for example, [17, 16, 28. In this paper, we generalize this strategy of globalization of Newton's method to solve the problem of finding singularities of vector fields defined on Riemannian manifolds. Until now, studies on the globalization strategies in Riemannian settings have been restricted to optimization problems, for example, the Newton's method with the hessian of the objective function updated by BFGS family, [5, 9, the Trust-Region methods, 29] and Levenberg-Marquardt methods [3, Chapter 8, Section 8.4.2]. To the best of our knowledge, a global analysis of the damped Newton's method for finding singu- 
larities of vector fields defined on Riemannian manifolds by using a linear search together with a merit function, whose particular case is to find local minimizers of real-valued functions defined on Riemannian manifolds, is novel here. Based on the idea presented in [30, Section 4] for nonlinear complementarity problem, we propose a damped Newton method in the Riemannian setting. Moreover, we shall show its global convergence to a singularity of the vector field preserving the same convergence rate of the classical Newton's method. We perform some numerical experiments for minimizing families of functions defined on cone of symmetric positive definite matrices which is one of Riemannian manifolds. Our experiments illustrate numerical performance of the proposed damped Newton method by linear search decreasing a merit function. The numerical results display that the damped Newton improves the behavior of the method when compared to the full step Newton method.

The remainder this paper is organized as follows. In Section 2 we present the notations and basic results used in the rest paper. In Section 3 we describe the global superlinear and quadratic convergence analysis of the damped Newton method. In Section 4 we display numerical experiments to verify the main theoretical results. Finally, we concludes the paper Section 5

\section{Basic Concepts and Auxiliary Results}

In this section we recall some notations, definitions and auxiliary results of Riemannian geometry used throughout the paper. Some basic concepts used here can be found in many introductory books on Riemannian geometry, for example, in [31 and 32. Let $\mathbb{M}$ be a finite dimensional Riemannian manifold, denote the tangent space of $\mathbb{M}$ at $p$ by $T_{p} \mathbb{M}$ and the tangent bundle of $\mathbb{M}$ by $T \mathbb{M}=$

$\bigcup_{p \in \mathbb{M}} T_{p} \mathbb{M}$. The corresponding norm associated to the Riemannian metric $\langle\cdot, \cdot\rangle$ is denoted by $\|\cdot\|$. The Riemannian distance between $p$ and $q$ in $\mathbb{M}$ is denoted by $d(p, q)$, which induces the original topology on $\mathbb{M}$, namely, $(\mathbb{M}, d)$ that is a complete metric space. The open ball of radius $r>0$ centred at $p$ is defined as $B_{r}(p):=\{q \in \mathbb{M}: d(p, q)<r\}$. Let $\Omega \subseteq \mathbb{M}$ be an open set and denote by 
$\mathcal{X}(\Omega)$ the space of differentiable vector fields on $\Omega$. Let $\nabla$ be the Levi-Civita connection associated to $(\mathbb{M},\langle\cdot, \cdot\rangle)$. The covariant derivative of $X \in \mathcal{X}(\Omega)$ determined by $\nabla$ defines at each $p \in \Omega$ a linear map $\nabla X(p): T_{p} \mathbb{M} \rightarrow T_{p} \mathbb{M}$ given by $\nabla X(p) v:=\nabla_{Y} X(p)$, where $Y$ is a vector field such that $Y(p)=v$. For $f: \mathbb{M} \rightarrow \mathbb{R}$, a twice-differentiable function the Riemannian metric induces the mappings $f \mapsto \operatorname{grad} f$ and $f \mapsto \operatorname{Hess} f$, which associate its gradient and hessian by the rules

$$
\langle\operatorname{grad} f, X\rangle:=d f(X), \quad\langle\operatorname{Hess} f X, X\rangle:=d^{2} f(X, X), \quad \forall X \in \mathcal{X}(\Omega),
$$

respectively, and the last equalities imply that $\operatorname{Hess} f X=\nabla_{X} \operatorname{grad} f$, for all $X \in \mathcal{X}(\Omega)$. For each $p \in \Omega$, the conjugate of a linear map $A_{p}: T_{p} \mathbb{M} \rightarrow$ $T_{p} \mathbb{M}$ is a linear map $A_{p}^{*}: T_{p} \mathbb{M} \rightarrow T_{p} \mathbb{M}$ defined by $\left\langle A_{p} v, u\right\rangle=\left\langle v, A_{p}^{*} u\right\rangle$, for all $u, v \in T_{p} M$. The norm of the linear map $A_{p}$ is defined by $\left\|A_{p}\right\|:=$ $\sup \left\{\left\|A_{p} v\right\|: v \in T_{p} \mathbb{M},\|v\|=1\right\}$. A vector field $V$ along a differentiable curve $\gamma$ in $M$ is said to be parallel if and only if $\nabla_{\gamma^{\prime}} V=0$. If $\gamma^{\prime}$ itself is parallel we say that $\gamma$ is a geodesic. The restriction of a geodesic to a closed bounded interval is called a geodesic segment. A geodesic segment joining $p$ to $q$ in $\mathbb{M}$ is said to be minimal if its length is equal to $d(p, q)$. If there exists a unique geodesic segment joining $p$ to $q$, then we denote it by $\gamma_{p q}$. For each $t \in[a, b], \nabla$ induces an isometric mapping, relative to $\langle\cdot, \cdot\rangle, P_{\gamma, a, t}: T_{\gamma(a)} \mathbb{M} \rightarrow T_{\gamma(t)} \mathbb{M}$ defined by $P_{\gamma, a, t} v=V(t)$, where $V$ is the unique vector field on $\gamma$ such that $\nabla_{\gamma^{\prime}(t)} V(t)=0$, and $V(a)=v$. This mapping is the so-called parallel transport along the geodesic segment $\gamma$ joining $\gamma(a)$ to $\gamma(t)$. Note also that $P_{\gamma, b_{1}, b_{2}} \circ P_{\gamma, a, b_{1}}=P_{\gamma, a, b_{2}}$ and $P_{\gamma, b, a}=P_{\gamma, a, b}^{-1}$. When there is no confusion we will consider the notation $P_{p q}$ instead of $P_{\gamma, a, b}$ in the case when $\gamma$ is the unique geodesic segment joining $p$ and $q$. A Riemannian manifold is complete if the geodesics are defined for any values of $t \in \mathbb{R}$. Hopf-Rinow's theorem asserts that every pair of points in a complete Riemannian manifold $\mathbb{M}$ can be joined by a (not necessarily unique) minimal geodesic segment. Due to the completeness of the Riemannian manifold $\mathbb{M}$, the exponential map $\exp _{p}: T_{p} \mathbb{M} \rightarrow \mathbb{M}$ can be given by $\exp _{p} v=\gamma(1)$, for each $p \in \mathbb{M}$, where $\gamma$ is the geodesic defined by its position $p$ and velocity $v$ 
at $p$. Let $p \in \mathbb{M}$, the injectivity radius of $\mathbb{M}$ at $p$ is defined by

$$
i_{p}:=\sup \left\{r>0: \exp _{p_{\left.\right|_{B_{r}\left(O_{p}\right)}}} \text { is a diffeomorphism }\right\},
$$

where $B_{r}\left(0_{p}\right):=\left\{v \in T_{p} \mathbb{M}:\left\|v-0_{p}\right\|<r\right\}$ and $0_{p}$ denotes the origin of the tangent plane $T_{p} \mathbb{M}$.

Remark 1. Let $\bar{p} \in \mathbb{M}$. The above definition implies that if $0<\delta<i_{\bar{p}}$, then $\exp _{\bar{p}} B_{\delta}\left(0_{\bar{p}}\right)=B_{\delta}(\bar{p})$. Moreover, for all $p \in B_{\delta}(\bar{p})$, there exists a unique geodesic segment $\gamma$ joining $p$ to $\bar{p}$, which is given by $\gamma_{p \bar{p}}(t)=\exp _{p}\left(t \exp _{p}^{-1} \bar{p}\right)$, for all $t \in[0,1]$.

Consider $p \in \mathbb{M}$ and $\delta_{p}:=\min \left\{1, i_{p}\right\}$. The quantity assigned to measure how fast the geodesic spread apart in $\mathbb{M}$ has been defined in [33] as

$$
\begin{aligned}
K_{p}:=\sup \left\{\frac{d\left(\exp _{q} u, \exp _{q} v\right)}{\|u-v\|}: q \in B_{\delta_{p}}(p), u, v \in T_{q} \mathbb{M}, u \neq v,\right. \\
\left.\|v\| \leq \delta_{p},\|u-v\| \leq \delta_{p}\right\} .
\end{aligned}
$$

Remark 2. In particular, when $u=0$ or more generally when $u$ and $v$ are on the same line through $0, d\left(\exp _{q} u, \exp _{q} v\right)=\|u-v\|$. Hence, $K_{p} \geq 1$, for all $p \in \mathbb{M}$. Moreover, when $\mathbb{M}$ has non-negative sectional curvature, the geodesics spread apart less than the rays [31, chapter 5], i.e., $d\left(\exp _{p} u, \exp _{p} v\right) \leq\|u-v\|$ and, in this case, $K_{p}=1$ for all $p \in \mathbb{M}$.

Let $X \in \mathcal{X}(\Omega)$ and $\bar{p} \in \Omega$. Assume that $0<\delta<\delta_{\bar{p}}$. Since $\exp _{\bar{p}} B_{\delta}\left(0_{\bar{p}}\right)=$ $B_{\delta}(\bar{p})$, there exists a unique geodesic joining each $p \in B_{\delta}(\bar{p})$ to $\bar{p}$. Moreover, using [22, equality 2.3$]$ we obtain

$$
X(p)=P_{\bar{p} p} X(\bar{p})+P_{\bar{p} p} \nabla X(\bar{p}) \exp _{\bar{p}}^{-1} p+d(p, \bar{p}) r(p), \quad \lim _{p \rightarrow \bar{p}} r(p)=0 .
$$

Let $p_{*} \in \mathbb{M}$. The following result establish that, if $\nabla X\left(p_{*}\right)$ is nonsingular there exists a neighborhood of $p_{*}$ which $\nabla X$ is nonsingular; see [24, Lemma 3.2].

Lemma 1. Let $X \in \mathcal{X}(\Omega)$ and $\bar{p} \in \mathbb{M}$. Assume that $\nabla X$ is continuous at $\bar{p}$, and $\nabla X(\bar{p})$ is nonsingular. Then, there exists $0<\hat{\delta}<\delta_{\bar{p}}$ such that $B_{\hat{\delta}}(\bar{p}) \subset \Omega$, and $\nabla X(p)$ is nonsingular for each $p \in B_{\hat{\delta}}(\bar{p})$. 
For any $X \in \mathcal{X}(\Omega)$ and $p \in \mathbb{M}$ such that $\nabla X(p)$ is nonsingular, the Newton's iterate mapping $N_{X}$ at $p$ is defined by

$$
N_{X}(p):=\exp _{p}\left(-\nabla X(p)^{-1} X(p)\right)
$$

In the folowing we present a result about the behavior of the Newton's iterate mapping near a singularity of $X$, whose proof can be found in [24, Lemma 3.3].

Lemma 2. Let $X: \Omega \rightarrow T \mathbb{M}$ a differentiable vector field and $p_{*} \in \mathbb{M}$. Assume that $X\left(p_{*}\right)=0, \nabla X$ is continuous at $p_{*}$ and $\nabla X\left(p_{*}\right)$ is nonsingular. Then, $\left.\lim _{p \rightarrow p_{*}}\left[d N_{X}(p), p_{*}\right) / d\left(p, p_{*}\right)\right]=0$.

The next result establishes superlinear convergence of the Newton's method; see [24, Theorem 3.1].

Theorem 1. Let $\Omega \subset \mathbb{M}$ be an open set, $X: \Omega \rightarrow T \mathbb{M}$ a differentiable vector field and $p_{*} \in \Omega$. Suppose that $p_{*}$ is a singularity of $X, \nabla X$ is continuous at $p_{*}$, and $\nabla X\left(p_{*}\right)$ is nonsingular. Then, there exists $\bar{\delta}>0$ such that, for all $p_{0} \in B_{\bar{\delta}}\left(p_{*}\right)$, the Newton sequence $p_{k+1}=\exp _{p_{k}}\left(-\nabla X\left(p_{k}\right)^{-1} X\left(p_{k}\right)\right)$, for all $k=0,1, \ldots$, is well-defined, contained in $B_{\bar{\delta}}\left(p_{*}\right)$, and converges superlinearly to $p_{*}$.

Let $X \in \mathcal{X}(\Omega)$ and $\bar{p} \in \Omega$. The map $\nabla X$ is locally Lipschitz continuous at $\bar{p}$ if and only if there exist a number $L>0$ and a $0<\delta_{L}<\delta_{p^{*}}$ such that $\left\|P_{p p^{*}} \nabla X(p)-\nabla X\left(p_{*}\right) P_{p p_{*}}\right\| \leq L d\left(p, p_{*}\right)$, for all $p \in B_{\delta_{L}}\left(p_{*}\right)$. We end this section with a result which, up to some minor adjustments, merges into [2, Theorem 4.4], see also [21, Theorem 7.1].

Theorem 2. Let $X: \Omega \rightarrow T \mathbb{M}$ be a continuously differentiable vector field, and $p_{*} \in \mathbb{M}$ a singularity of $X$. Suppose that $\nabla X$ is locally Lipschitz continuous at $p_{*}$ with constant $L>0$, and $\nabla X\left(p_{*}\right)$ is nonsingular. Then, there exists a $r>0$ such that the sequence $p_{k+1}=\exp _{p_{k}}\left(-\nabla X\left(p_{k}\right)^{-1} X\left(p_{k}\right)\right)$, for all $k=0,1, \ldots$, starting in $p_{0} \in B_{r}\left(p_{*}\right) \backslash\left\{p_{*}\right\}$, is well-defined, contained $B_{r}\left(p_{*}\right)$, converges to $p_{*}$, and there holds

$$
d\left(p_{*}, p_{k+1}\right) \leq \frac{L K_{p_{*}}^{2}\left\|\nabla X\left(p^{*}\right)^{-1}\right\|}{2\left[K_{p_{*}}-d\left(p_{0}, p_{*}\right) L\left\|\nabla X\left(p^{*}\right)^{-1}\right\|\right]} d\left(p_{*}, p_{k}\right)^{2}, \quad k=0,1, \ldots
$$




\section{Damped Newton Method}

In this section we consider the problem of finding a singularity of a differentiable vector field. The formal statement of the problem is as follows: Find a point $p \in \mathbb{M}$ such that

$$
X(p)=0,
$$

where $X: \Omega \subseteq \mathbb{M} \rightarrow T \mathbb{M}$ is a differentiable vector field. The Newton's method applied to this problem has local superlinear convergence, whenever the covariant derivative in the singularity of $X$ is non-singular, see [24]. Additionally, if the covariant derivative is Lipschitz continous at singularity, then the method has $Q$-quadratic convergence, see [21. In order to globalize the Newton's method keeping the same properties aforementioned, we will use the same idea of the Euclidean setting by introducing a linear search strategy. This modification of Newton's method called damped Newton's method performs a linear search decreasing the merit function $\varphi: \mathbb{M} \rightarrow \mathbb{R}$,

$$
\varphi(p)=\frac{1}{2}\|X(p)\|^{2},
$$

in order to reach the superlinear convergence region of the Newton's method. In the following we present the formal description of the damped Newton's algorithm.

\section{Damped Newton Method}

Step 0. Choose a scalar $\sigma \in(0,1 / 2)$, take an initial point $p_{0} \in \mathbb{M}$, and set $k=0$;

Step 1. Compute search direction $v_{k} \in T_{p_{k}} \mathbb{M}$ as a solution of the linear equation

$$
X\left(p_{k}\right)+\nabla X\left(p_{k}\right) v=0 .
$$

If $v_{k}$ exists, go to Step 2. Otherwise, set the search direction $v_{k}=$ $-\operatorname{grad} \varphi\left(p_{k}\right)$, where $\varphi$ is defined by (5), i.e.,

$$
v_{k}=-\nabla X\left(p_{k}\right)^{*} X\left(p_{k}\right) .
$$


If $v_{k}=0$, stop;

Step 2. Compute the stepsize by the rule

$$
\begin{aligned}
\alpha_{k}:=\max \left\{2^{-j}: \varphi\left(\exp _{p_{k}}\left(2^{-j} v_{k}\right)\right) \leq \varphi\left(p_{k}\right)+\right. \\
\left.\sigma 2^{-j}\left\langle\operatorname{grad} \varphi\left(p_{k}\right), v_{k}\right\rangle, j \in \mathbb{N}\right\} ;
\end{aligned}
$$

and set the next iterated as

$$
p_{k+1}:=\exp _{p_{k}}\left(\alpha_{k} v_{k}\right)
$$

Step 3. Set $k \leftarrow k+1$ and go to Step 1 .

We remark that in Step 1 we resort directly to the steepest descent step of $\varphi$ as a safeguard, but only in those cases when the Newtonian direction in (6) does not exist. This idea has been used, in Euclidian context, for nonlinear complementarity problems, see for example [30] and [26, Chap. 5, Sec. 5.1.1].

\subsection{Preliminaries}

In this subsection we present some preliminaries results in order to ensure well-definition and convergence of the sequence generated by the damped Newton's method. We begin with an useful result for establishing the well-defined sequence.

Lemma 3. Let $p \in \Omega$ such that $X(p) \neq 0$. Assume that $v=-\nabla X(p)^{*} X(p)$ or that $v$ is a solution of the following equation

$$
X(p)+\nabla X(p) v=0 .
$$

If $v \neq 0$, then $v$ is a descent direction for $\varphi$ from $p$, i.e., $\langle\operatorname{grad} \varphi(p), v\rangle<0$.

Proof. First we assume that $v$ satisfies 10 . Since $\operatorname{grad} \varphi(p)=\nabla X(p)^{*} X(p)$, there is $\langle\operatorname{grad} \varphi(p), v\rangle=\langle X(p), \nabla X(p) v\rangle$. It follows from $X(p) \neq 0$ and $v$ satisfying 10 that $\langle\operatorname{grad} \varphi(p), v\rangle=-\|X(p)\|^{2}<0$, which implies that $v$ is a descent direction for $\varphi$ from $p$. Now, assume that $v=-\nabla X(p)^{*} X(p)$. Thus $\langle\operatorname{grad} \varphi(p), v\rangle=-\left\|\nabla X(p)^{*} X(p)\right\|^{2}<0$ and $v$ is also a descent direction for $\varphi$ from $p$. 
Under suitable assumptions the following result guarantees that the damped Newton's method, after a finite quantity of iterates, reduces to the classical iteration of Newton's method.

Lemma 4. Let $\bar{p} \in \mathbb{M}$ satisfy $X(\bar{p})=0$. If $\nabla X$ and $\nabla X(\bar{p})$ are continuous at $\bar{p}$ and nonsingular respectively, then there exists $0<\hat{\delta}<\delta_{\bar{p}}$ such that $B_{\hat{\delta}}(\bar{p}) \subset \Omega$, $\nabla X(p)$ is nonsingular for each $p \in B_{\hat{\delta}}(\bar{p})$ and

$$
\lim _{p \rightarrow \bar{p}} \frac{\varphi\left(N_{X}(p)\right)}{\|X(p)\|^{2}}=0
$$

As a consequence, there exists a $\delta>0$ such that, for all $\sigma \in(0,1 / 2)$ and $\delta<\hat{\delta}$ there holds

$$
\left.\varphi\left(N_{X}(p)\right) \leq \varphi(p)\right)+\sigma\left\langle\operatorname{grad} \varphi(p),-\nabla X(p)^{-1} X(p)\right\rangle, \quad \forall p \in B_{\delta}(\bar{p}) .
$$

Proof. By Lemma 1 there exists $0<\hat{\delta}<\delta_{\bar{p}}$ such that $B_{\hat{\delta}}(\bar{p}) \subset \Omega$ and $\nabla X(p)$ is nonsingular for each $p \in B_{\hat{\delta}}(\bar{p})$. Define $v_{p}=-\nabla X(p)^{-1} X(p)$, for all $p \in$ $B_{\hat{\delta}}(\bar{p})$. Since $X(\bar{p})=0$ and $\nabla X$ is continuous and nonsingular at $\bar{p}$, there is $\lim _{p \rightarrow \bar{p}} v_{p}=0$. Moreover, it follows from (2), the isometry of the parallel transport and $\left\|\exp _{\bar{p}}^{-1} \exp _{p}\left(v_{p}\right)\right\|=d\left(\exp _{p}\left(v_{p}\right), \bar{p}\right)$ that

$$
\begin{gathered}
\varphi\left(N_{X}(p)\right)=\frac{1}{2}\left\|X\left(\exp _{p}\left(v_{p}\right)\right)-P_{\bar{p} \exp _{p}\left(v_{p}\right)} X(\bar{p})\right\|^{2} \leq \\
{\left[\|\nabla X(\bar{p})\|+\left\|r\left(\exp _{p}\left(v_{p}\right)\right)\right\|\right]^{2} d^{2}\left(\exp _{p}\left(v_{p}\right), \bar{p}\right) .}
\end{gathered}
$$

Hence, after some simple algebraic manipulations and from the last inequality we obtain for all $p \in B_{\hat{\delta}}(\bar{p}) \backslash\{\bar{p}\}$,

$$
\frac{\varphi\left(\exp _{p}\left(v_{p}\right)\right)}{\|X(p)\|^{2}} \leq\left[\|\nabla X(\bar{p})\|+\left\|r\left(\exp _{p}\left(v_{p}\right)\right)\right\|\right]^{2} \frac{d^{2}\left(\exp _{p}\left(v_{p}\right), \bar{p}\right)}{d^{2}(p, \bar{p})} \frac{d^{2}(p, \bar{p})}{\|X(p)\|^{2}} .
$$

On the other hand, owing that $X(\bar{p})=0$ and $\nabla X(\bar{p})$ is nonsingular, it is easy to see that

$$
\begin{array}{r}
\exp _{\bar{p}}^{-1} p=-\nabla X(\bar{p})^{-1}\left[P_{p \bar{p}} X(p)-X(\bar{p})-\nabla X(\bar{p}) \exp _{\bar{p}}^{-1} p\right]+ \\
\nabla X(\bar{p})^{-1} P_{p \bar{p}} X(p) .
\end{array}
$$


Using again (2) and that $\nabla X(\bar{p})$ is nonsingular, we conclude that there exists $0<\bar{\delta}<\delta_{\bar{p}}$ such that

$$
\left\|\nabla X(\bar{p})^{-1}\left[P_{p \bar{p}} X(p)-X(\bar{p})-\nabla X(\bar{p}) \exp _{\bar{p}}^{-1} p\right]\right\| \leq \frac{1}{2} d(p, \bar{p}), \quad \forall p \in B_{\bar{\delta}}(\bar{p}) .
$$

Thus, combining (14) with last inequality and considering that $d(\bar{p}, p)=\left\|\exp _{\bar{p}}^{-1} p\right\|$ we obtain that $d(\bar{p}, p) \leq d(p, \bar{p}) / 2+\left\|\nabla X(\bar{p})^{-1} P_{p \bar{p}} X(p)\right\|$, for all $p \in B_{\bar{\delta}}(\bar{p})$, which implies that $d^{2}(\bar{p}, p) \leq\left[2\left\|\nabla X(\bar{p})^{-1}\right\|\right]^{2}\|X(p)\|^{2}$, for all $p \in B_{\bar{\delta}}(\bar{p})$. Hence, letting $\tilde{\delta}=\min \{\hat{\delta}, \bar{\delta}\}$ we obtain from $(13)$ that for all $p \in B_{\tilde{\delta}}(\bar{p}) \backslash\{\bar{p}\}$,

$$
\frac{\varphi\left(\exp _{p}\left(v_{p}\right)\right)}{\|X(p)\|^{2}} \leq\left[2\left\|\nabla X(\bar{p})^{-1}\right\|\left(\|\nabla X(\bar{p})\|+\left\|r\left(\exp _{p}\left(v_{p}\right)\right)\right\|\right)\right]^{2} \frac{d^{2}\left(\exp _{p}\left(v_{p}\right), \bar{p}\right)}{d^{2}(p, \bar{p})}
$$

Therefore, it follows from Lemma 2 and $\lim _{p \rightarrow \bar{p}} r\left(\exp _{p}\left(v_{p}\right)\right)=0$ that the equality (11) by taking limit, as $p$ goes to $\bar{p}$, in the latter inequality. For proving [12], we first use 111) for concluding that there exists a $\delta>0$ such that, $\delta<\hat{\delta}$ and for $\sigma \in(0,1 / 2)$ there is

$$
\varphi\left(N_{X}(p)\right) \leq \frac{1-2 \sigma}{2}\|X(p)\|^{2}, \quad \forall p \in B_{\delta}(\bar{p}) .
$$

Taking into account that $\operatorname{grad} \varphi(p)=\nabla X(p)^{*} X(p)$ we can conclude directly that $\left\langle\operatorname{grad} \varphi(p),-\nabla X(p)^{-1} X(p)\right\rangle=-\|X(p)\|^{2}$, then the last inequality is equivalent to 12 and then the proof is complete.

In the next result we show that whenever the vector field is continuous with nonsingular covariant derivative at a singularity, there exists a neighborhood which is invariant by the Newton's iterate mapping associated.

Lemma 5. Let $\bar{p} \in \mathbb{M}$ such that $X(\bar{p})=0$. If $\nabla X$ is continuous at $\bar{p}$ and $\nabla X(\bar{p})$ is nonsingular, then there exists $0<\hat{\delta}<\delta_{\bar{p}}$ such that $B_{\hat{\delta}}(\bar{p}) \subset \Omega$ and $\nabla X(p)$ is nonsingular for each $p \in B_{\hat{\delta}}(\bar{p})$. Moreover, $N_{X}(p) \in B_{\hat{\delta}}(\bar{p})$, for all $p \in B_{\hat{\delta}}(\bar{p})$.

Proof. It follows from Lemma 1 that there exists $0<\hat{\delta}<\delta_{\bar{p}}$ such that $B_{\hat{\delta}}(\bar{p}) \subset \Omega$ and $\nabla X(p)$ is nonsingular for each $p \in B_{\hat{\delta}}(\bar{p})$. Thus, shirking $\hat{\delta}$ if necessary, we can use Lemma 2 to conclude that $d\left(N_{X}(p), \bar{p}\right)<d(p, \bar{p}) / 2$, for all $p \in B_{\hat{\delta}}\left(p_{*}\right)$, which implies the last statement of the lemma. 


\subsection{Convergence Analysis}

In this subsection we establish our main result. We shall prove a result on the global convergence of the damped Newton's method preserving the fast convergence rates of the Newton's one. We begin by proving the well-definition of the sequence generated by the damped Newton's method.

Lemma 6. The sequence $\left\{p_{k}\right\}$ generated by the damped Newton's method is well-defined.

Proof. If $v_{0} \neq 0$ and $X\left(p_{0}\right) \neq 0$, then by using Lemma 3 we can conclude that $v_{0}$ satisfying (6) or (7) is a descent direction for $\varphi$ from $p_{0}$. Hence, by using ordinary argument we conclude that $\alpha_{0}$ in $(8)$ is well-defined, and $p_{1}$ in $(9)$ is also well-defined. Therefore, by using induction argument we can prove that the sequence $\left\{p_{k}\right\}$ is well-defined.

Note that, if the sequence generated by the damped Newton's method is finite, then the last iterate is a solution of (4) or a critical point of $\varphi$ defined in (5). Thus, we can assume that $\left\{p_{k}\right\}$ is infinite, $v_{k} \neq 0$ and $X\left(p_{k}\right) \neq 0$, for all $k=0,1, \ldots$

Theorem 3. Let $X: \Omega \subseteq \mathbb{M} \rightarrow T \mathbb{M}$ be a differentiable vector field. Assume that $\left\{p_{k}\right\}$ generated by the Damped Newton's Method has an accumulation point $\bar{p} \in \Omega$ such that $\nabla X$ is continuous at $\bar{p}$ with nonsingular $\nabla X(\bar{p})$. Then, $\left\{p_{k}\right\}$ converges superlinearly to the singularity $\bar{p}$ of $X$. Moreover, the convergence rate is quadratic provided that $\nabla X$ is locally Lipschitz continuous at $\bar{p}$.

Proof. We begin to show that $\bar{p}$ is a singularity of $X$. As aforementioned, we can assume that $\left\{p_{k}\right\}$ is infinite, $\operatorname{grad} \varphi\left(p_{k}\right) \neq 0$ and $X\left(p_{k}\right) \neq 0$, for all $k=0,1, \ldots$ Hence, (8) and (9) imply that

$$
\begin{aligned}
\varphi\left(p_{k}\right)-\varphi\left(p_{k+1}\right) \geq-\sigma \alpha_{k}\left\langle\operatorname{grad} \varphi\left(p_{k}\right), v_{k}\right\rangle \\
\qquad= \begin{cases}\sigma \alpha_{k}\left\|X\left(p_{k}\right)\right\|^{2}>0, & \text { if } v_{k} \text { satisfies (6); } \\
\sigma \alpha_{k}\left\|\operatorname{grad} \varphi\left(p_{k}\right)\right\|^{2}>0, & \text { else. }\end{cases}
\end{aligned}
$$


Then $\left\{\varphi\left(p_{k}\right)\right\}$ is strictly decreasing and, bounded below by zero which results in convergence of the sequence. Hence, taking the limite in this latter inequality we obtain $\lim _{k \rightarrow \infty}\left[\alpha_{k}\left\langle\operatorname{grad} \varphi\left(p_{k}\right), v_{k}\right\rangle\right]=0$. Let $\left\{p_{k_{j}}\right\}$ be a subsequence of $\left\{p_{k}\right\}$ such that $\lim _{k_{j} \rightarrow+\infty} p_{k_{j}}=\bar{p}$. Thus, we have

$$
\lim _{k_{j} \rightarrow \infty}\left[\alpha_{k_{j}}\left\langle\operatorname{grad} \varphi\left(p_{k_{j}}\right), v_{k_{j}}\right\rangle\right]=0 .
$$

Owing that $\nabla X$ is continuous at $\bar{p}$ and $\nabla X(\bar{p})$ is nonsingular, by using Lemma 1 . we can also assume that $\nabla X\left(p_{k_{j}}\right)$ is nonsingular, for all $j=0,1, \ldots$ Hence, (6) has a solution and then for $j=0,1, \ldots$ we have

$$
v_{k_{j}}=-\nabla X\left(p_{k_{j}}\right)^{-1} X\left(p_{k_{j}}\right), \quad\left\langle\operatorname{grad} \varphi\left(p_{k_{j}}\right), v_{k_{j}}\right\rangle=-\left\|X\left(p_{k_{j}}\right)\right\|^{2} .
$$

To analyse the consequences of 15 we consider the following two possible cases:
a) $\liminf \operatorname{in}_{j \rightarrow \infty} \alpha_{k_{j}}>0$,
b) $\liminf f_{j \rightarrow \infty} \alpha_{k_{j}}=0$.

First we assume item a) holds. From (15), passing onto a further subsequence if necessary, we can assume that

$$
\lim _{j \rightarrow \infty}\left\langle\operatorname{grad} \varphi\left(p_{k_{j}}\right), v_{k_{j}}\right\rangle=0 .
$$

Taking the limit in the latter equality, as $j$ goes to infinity, and considering that $\lim _{j \rightarrow+\infty} p_{k_{j}}=\bar{p}$ and $X$ is continuous at $\bar{p}$, we conclude from 17) and 16 that $X(\bar{p})=0$ what means $\bar{p}$ is a singularity of $X$. Now, we assume item b) holds. Hence, given $s \in \mathbb{N}$ we can take $j$ large enough such that $\alpha_{k_{j}}<2^{-s}$. Thus $2^{-s}$ does not satisfies the Armijo's condition (8), i.e.,

$$
\varphi\left(\exp _{p_{k_{j}}}\left(2^{-s} v_{k}\right)\right)>\varphi\left(p_{k_{j}}\right)+\sigma 2^{-s}\left\langle\operatorname{grad} \varphi\left(p_{k_{j}}\right), v_{k_{j}}\right\rangle
$$

Letting $j$ goes to infinity, considering that the exponential mapping is continuous, $\lim _{j \rightarrow+\infty} p_{k_{j}}=\bar{p}$ and due to $\nabla X$ and $X$ be continuous at $\bar{p}$, it follows from (16) and the last inequality that

$$
\varphi\left(\exp _{\bar{p}}\left(2^{-s} \bar{v}\right)\right)>\varphi(\bar{p})+\sigma 2^{-s}\langle\operatorname{grad} \varphi(\bar{p}), \bar{v}\rangle,
$$

where $\bar{v}=-\nabla X(\bar{p})^{-1} X(\bar{p})$. Hence, we obtain $\left[\varphi\left(\exp _{\bar{p}}\left(2^{-s} \bar{v}\right)\right)-\varphi(\bar{p})\right] / 2^{-s} \geq$ $\sigma\langle\operatorname{grad} \varphi(\bar{p}), \bar{v}\rangle$. Therefore, letting $s$ goes to infinity we can conclude that 
$\langle\operatorname{grad} \varphi(\bar{p}), \bar{v}\rangle \geq \sigma\langle\operatorname{grad} \varphi(\bar{p}), \bar{v}\rangle$, or equivalently $\|X(\bar{p})\|^{2} \leq \sigma \| X\left(\bar{p} \|^{2}\right.$. Thus, owing $\sigma \in(0,1 / 2)$ we have $\|X(\bar{p})\|=0$ which implies that $\bar{p}$ is singularity of $X$.

We proceed to prove that there exists $k_{0}$ such that $\alpha_{k}=1$, for all $k \geq k_{0}$. Since $\nabla X(\bar{p})$ is nonsingular and $X(\bar{p})=0$, the Lemma 1 and Lemma 5 imply that there exists $\hat{\delta}>0$ such that $\nabla X(p)$ is nonsingular and $N_{X}(p) \in B_{\delta}(\bar{p})$, for all $p \in B_{\delta}(\bar{p})$ and all $\delta \leq \hat{\delta}$. Since $\bar{p}$ is a cluster point of $\left\{p_{k}\right\}$, there exits $k_{0}$ such that $p_{k_{0}} \in B_{\hat{\delta}}(\bar{p})$ (shirking $\hat{\delta}$ if necessary). It follows from Lemma 4 that $\left.\varphi\left(N_{X}\left(p_{k_{0}}\right)\right) \leq \varphi\left(p_{k_{0}}\right)\right)+\sigma\left\langle\operatorname{grad} \varphi\left(p_{k_{0}}\right), v_{k_{0}}\right\rangle$, with $v_{k_{0}}=-\nabla X\left(p_{k_{0}}\right)^{-1} X\left(p_{k_{0}}\right)$. Hence, it follows from the last inequality, (3) and (8) that $\alpha_{k_{0}}=1$. By (9) we can conclude that $p_{k_{0}+1}=N_{X}\left(p_{k_{0}}\right)$ which implies $p_{k_{0}+1} \in B_{\hat{\delta}}(\bar{p})$ because of $N_{X}\left(p_{k_{0}}\right) \in B_{\hat{\delta}}(\bar{p})$. Then, an induction step is completely analogous, yielding

$$
\alpha_{k}=1, \quad p_{k+1}=N_{X}\left(p_{k}\right) \in B_{\hat{\delta}}(\bar{p}), \quad \forall k \geq k_{0} .
$$

In order to obtain supetlinear convergence of the entire sequence $\left\{p_{k}\right\}$, let $\bar{\delta}>0$ be given by Theorem 1. Thus, making $\hat{\delta}$ smaller if necessary so that $\hat{\delta}<\bar{\delta}$, we can apply Theorem 1 to conclude from 18 that $\left\{p_{k}\right\}$ converges superlinearly to $\bar{p}$.

To prove that $\left\{p_{k}\right\}$ converges quadratically to $\bar{p}$, we first make $\hat{\delta}<r$, where $r$ is given by Theorem 2 . Since $\nabla X$ is locally Lipschitz continuous at $\bar{p}$, the result follows from the combination of (18) and Theorem 2 .

\section{Numerical Experiments}

In this section, we shall give some numerical experiments to illustrate the performance of the damped Newton method for minimizing two families of functions defined on the cone of symmetric positive definite matrices. A particular instance of one of these families has application in robotics. Before present our numerical experiment, we need to introduce some concepts. Let $\mathbb{P}^{n}$ be the set of symmetric matrices of order $n \times n$ and $\mathbb{P}_{++}^{n}$ be the cone of symmetric positive definite matrices. Following Rothaus [34, let $\mathbb{M}:=\left(\mathbb{P}_{++}^{n},\langle\rangle,\right)$ be the 
Riemannian manifold endowed with the Riemannian metric defined by

$$
\langle U, V\rangle=\operatorname{tr}\left(V \psi^{\prime \prime}(P) U\right)=\operatorname{tr}\left(V P^{-1} U P^{-1}\right), P \in \mathbb{M}, \quad U, V \in T_{P} \mathbb{M} \approx \mathbb{P}^{n}
$$

where $\operatorname{tr} P$ denotes the trace of $P$. Then, the exponential mapping is given by

$$
\exp _{P} V=P^{1 / 2} e^{\left(P^{-1 / 2} V P^{-1 / 2}\right)} P^{1 / 2}, \quad P \in \mathbb{M}, \quad V \in T_{P} \mathbb{M} \approx \mathbb{P}^{n} .
$$

Let $X$ and $Y$ be vector fields in $\mathbb{M}$. Then, by using [32, Theorem 1.2, page 28], we can prove that the Levi-Civita connection of $\mathbb{M}$ is given by

$$
\nabla_{Y} X(P)=X^{\prime} Y-\frac{1}{2}\left[Y P^{-1} X+X P^{-1} Y\right]
$$

where $P \in \mathbb{M}$, and $X^{\prime}$ denotes the Euclidean derivative of $X$. Therefore, it follows from 19 and 21) that the Riemannian gradient and hessian of a twicedifferentiable function $f: \mathbb{M} \rightarrow \mathbb{R}$ are respectively given by:

$$
\begin{aligned}
\operatorname{grad} f(P)=P f^{\prime}(P) P, \quad \operatorname{Hess} f(P) V=P f^{\prime \prime}(P) V P+ & \\
& \frac{1}{2}\left[V f^{\prime}(P) P+P F^{\prime}(P) V\right],
\end{aligned}
$$

for all $V \in T_{P} \mathbb{M}$, where $f^{\prime}(P)$ and $f^{\prime \prime}(P)$ are the Euclidean gradient and hessian of $f$ at $P$, respectively. In this case, by using $(20)$, the Newton's iteration for finding $P \in \mathbb{M}$ such that $\operatorname{grad} f(P)=0$ is given by

$$
P_{k+1}=P_{k}^{1 / 2} e^{P_{k}^{-1 / 2} V_{k} P_{k}^{-1 / 2}} P_{k}^{1 / 2}, \quad k=0,1, \ldots,
$$

where, by using again the equalities in $(22), V_{k}$ is the unique solution of the Newton's linear equation

$$
P_{k} f^{\prime \prime}\left(P_{k}\right) V_{k} P_{k}+\frac{1}{2}\left[V_{k} f^{\prime}\left(P_{k}\right) P_{k}+P_{k} f^{\prime}\left(P_{k}\right) V_{k}\right]=-P_{k} f^{\prime}\left(P_{k}\right) P_{k},
$$

which corresponds to 6 for $X=\operatorname{grad} f$. Now, we are going to present concrete examples for (23) and (24). Let $i=1,2$ and $f_{i}: \mathbb{P}_{++}^{n} \rightarrow \mathbb{R}$ be defined, respectively, by

$$
f_{1}(P)=\mathrm{a}_{1} \ln \operatorname{det} P+\mathrm{b}_{1} \operatorname{tr} P^{-1}, \quad f_{2}(P)=\mathrm{a}_{2} \ln \operatorname{det} P-\mathrm{b}_{2} \operatorname{tr} P,
$$


where $\mathrm{b}_{1} / \mathrm{a}_{1}>0, \mathrm{a}_{2} / \mathrm{b}_{2}>0$, and $\operatorname{det} P$ denotes the determinant of $P$, respectively. In particular, the function $f_{2}$ can be associated to the optimal dextrous hand grasping problem in robotics, see [35, 36, 37.

Then, for each $i=1,2$, the Euclidean gradient and hessian of $f_{i}$ are given, respectively, by

$$
\begin{aligned}
& f_{1}^{\prime}(P)=\mathrm{a}_{1} P^{-1}-\mathrm{b}_{1} P^{-2}, \\
& f_{1}^{\prime \prime}(P) V=\mathrm{b}_{1}\left(P^{-1} V P^{-2}+P^{-2} V P^{-1}\right)-\mathrm{a}_{1} P^{-1} V P^{-1}, \\
& f_{2}^{\prime}(P)=\mathrm{a}_{2} P^{-1}-\mathrm{b}_{2} I, \quad f_{2}^{\prime \prime}(P) V_{k}=-\mathrm{a}_{2} P^{-1} V P^{-1},
\end{aligned}
$$

where $I$ denotes the $n \times n$ identity matrix. It follows from $(22),(25),(26)$ and (27) that

$$
\operatorname{grad} f_{1}(P)=\mathrm{a}_{1} P-\mathrm{b}_{1} I, \quad \operatorname{grad} f_{2}(P)=\mathrm{a}_{2} P-\mathrm{b}_{2} P^{2} .
$$

From the last two equalities, we can conclude that the global minimizer of $f_{i}$, for each $i=1,2$ are $P_{1}^{*}=\mathrm{b}_{1} / \mathrm{a}_{1} I$ and $P_{2}^{*}=\mathrm{a}_{2} / \mathrm{b}_{2} I$, respectively. Our task is to execute explicitly the Damped Newton method to find the global minimizer of $f_{i}$, for each $i=1,2$. For this purpose, consider $\varphi_{i}: \mathbb{P}_{++}^{n} \rightarrow \mathbb{R}$ given by $\varphi_{i}(P)=1 / 2\left\|\operatorname{grad} f_{i}(P)\right\|^{2}, i=1,2$. Thus, by using 28 we conclude that

$$
\operatorname{grad} \varphi_{1}(P)=\mathrm{ab} I-\mathrm{b}_{1}^{2} P^{-1}, \quad \operatorname{grad} \varphi_{2}(P)=d^{2} P^{3}-\mathrm{a}_{2} \mathrm{~b}_{2} P^{2} .
$$

By combining (19), 20) and 28), after some calculations we obtain the following equalities

$$
\begin{aligned}
& \varphi_{1}\left(\exp _{P} V\right)=\frac{1}{2} \operatorname{tr}\left(\mathrm{a}_{1} I-\mathrm{b}_{1}\left(P^{1 / 2} e^{P^{-1 / 2} V P^{-1 / 2}} P^{1 / 2}\right)^{-1}\right)^{2}, \\
& \varphi_{2}\left(\exp _{P} V\right)=\frac{1}{2} \operatorname{tr}\left(\mathrm{a}_{2} I-\mathrm{b}_{2} P^{1 / 2} e^{P^{-1 / 2} V P^{-1 / 2}} P^{1 / 2}\right)^{2} .
\end{aligned}
$$

Finally, by using 223, 24, definition of $f_{1}$ in 25, 26, left hand sides of 28 and 29 and 30 we give the damped Newton method, for finding the global minimizer of $f_{1}$. The formal algorithm to find the global minimizer of function $f_{2}$ will not be presented here because it can be obtained similarly by using [23, 
(24), the definition of $f_{2}$ in (25), (27), right hand sides of (28) and 29$)$ and (31). The Damped Newton algorithim for the matrix cone is given as follows.

\section{Damped Newton Method in $\mathbb{P}_{++}^{n}\left(\mathrm{DNM}-\mathbb{P}_{++}^{n}\right)$}

Step 0. Choose a scalar $\sigma \in(0,1 / 2)$, take an initial point $P_{0} \in \mathbb{M}$, and set $k=0$

Step 1. Compute search direction $V_{k}$, as a solution of the linear equation

$$
P_{k} V_{k}+V_{k} P_{k}=2\left(P_{k}^{2}-a / b P_{k}^{3}\right) .
$$

If $V_{k}$ exists go to Step 2. Othewise, put $V_{k}=-\operatorname{grad} \varphi_{1}\left(p_{k}\right)$, i.e,

$$
V_{k}=\mathrm{b}_{1}^{2} P_{k}^{-1}-\mathrm{ab} I
$$

If $V_{k}=0$, stop;

Step 2. Compute the stepsize by the rule

$$
\begin{array}{r}
\alpha_{k}:=\max \left\{2^{-j}: \frac{1}{2} \operatorname{tr}\left(\mathrm{a}_{1} I-\mathrm{b}_{1}\left(P_{k}^{1 / 2} e^{\left.\left.2^{-j} P_{k}^{-1 / 2} V P_{k}^{-1 / 2} P_{k}^{1 / 2}\right)^{-1}\right)^{2} \leq}\right.\right.\right. \\
\left.\left(\frac{1}{2}-\sigma 2^{-j}\right) \operatorname{tr}\left(\mathrm{a}_{1} I-\mathrm{b}_{1} P_{k}^{-1}\right)^{2}\right\}
\end{array}
$$

and set the next iterated as

$$
P_{k+1}:=P_{k}^{1 / 2} e^{\alpha_{k} P_{k}^{-1 / 2} V_{k} P_{k}^{-1 / 2}} P_{k}^{1 / 2}
$$

Step 3. Set $k \leftarrow k+1$ and go to Step 1 .

Although the domain of $f_{1}$ is a subset of the symmetric matrix set, namely, $\mathbb{P}_{++}^{n}$, equality $(9)$ shows that DNM- $\mathbb{P}_{++}^{n}$ generates only feasible points without using projections or any other procedure to remain the feasibility. Hence, problem of minimizing $f_{1}$ onto $\mathbb{P}_{++}^{n}$ can be seen as unconstrained Riemannian 
optimization problem. Note that, in this case, the equation 32 always has unique solution $V_{k} \in T_{P_{k}} \mathbb{P}_{++}^{n}$, see [38, Th. 8.2.1]. Consequently the direction $V_{k}$ in (33) does not play any role here. Thus, in this case, we can compare the Newton and damped Newton methods in number of iterations, function evaluation and CPU time to reach the solution. The Newton method is formally described as follow.

\section{Newton Method in $\mathbb{P}_{++}^{n}\left(\mathrm{NM}-\mathbb{P}_{++}^{n}\right)$}

Step 0. Take an initial point $P_{0} \in \mathbb{M}$, and set $k=0$;

Step 1. Compute search direction $V_{k}$ as a solution of the linear equation

$$
P_{k} V_{k}+V_{k} P_{k}=2\left(P_{k}^{2}-\mathrm{a}_{1} / \mathrm{b}_{1} P_{k}^{3}\right)
$$

Step 2. Compute the next iterated by

$$
P_{k+1}:=P_{k}^{1 / 2} e^{P_{k}^{-1 / 2} V_{k} P_{k}^{-1 / 2}} P_{k}^{1 / 2}
$$

Step 3. Set $k \leftarrow k+1$ and go to Step 1 .

We have implemented the above two algorithms by using MATLAB R2015b. The experiments are performed on an Intel Core 2 Duo Processor $2.26 \mathrm{GHz}$, 4 GB of RAM, and OSX operating system. We compare the iterative behavior of NM- $\mathbb{P}_{++}^{n}$ and DNM- $\mathbb{P}_{++}^{n}$ applied to $f_{1}$ and $f_{2}$ with randomly chosen initial guesses. In all experiments the stop criterion at the iterate $P_{k} \in \mathbb{P}_{++}^{n}$ is $\left\|\operatorname{grad} f\left(P_{k}\right)\right\| \leq 10^{-8}$ where $\|\cdot\|$ is norm associated to the metric given by (19). All codes are freely available at http://www2.uesb.br/professor/ mbortoloti/wp-content/uploads/2018/03/MATLAB_codes.zip. We run the above two algorithms in a huge number of problems by varying the dimensions and parameters $\mathrm{b}_{1} / \mathrm{a}_{1}$ and $\mathrm{b}_{2} / \mathrm{a}_{2}$ in functions $f_{1}$ and $f_{2}$, respectively. In general, DNM- $\mathbb{P}_{++}^{n}$ is superior to NM- $\mathbb{P}_{++}^{n}$ in number of iterations and CPU time. 


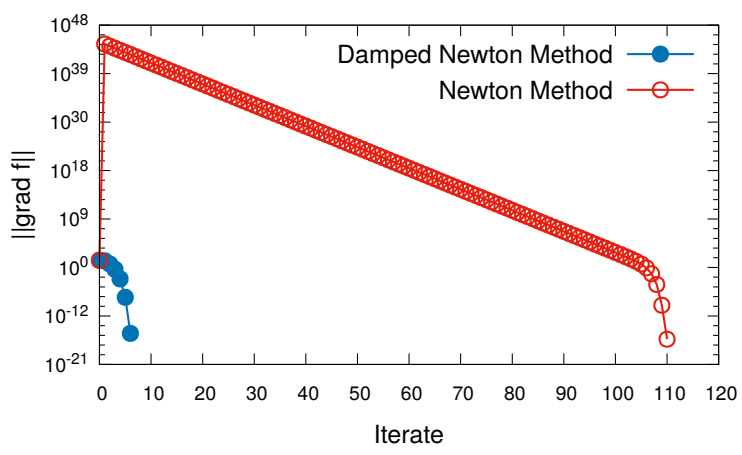

(a) $f_{1}$ with $\mathrm{b}_{1} / \mathrm{a}_{1}=0.1$ and $n=1000$.

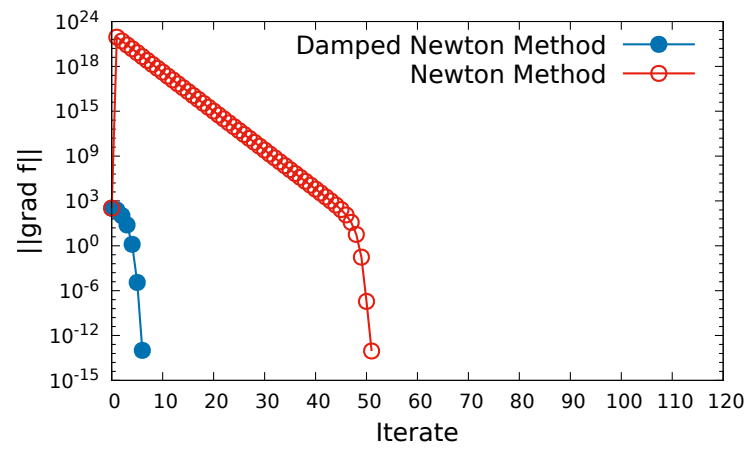

(b) $f_{2}$ with $\mathrm{b}_{2} / \mathrm{a}_{2}=0.002$ and $n=1000$.

As we can see in figures (a) and (b) NM- $\mathbb{P}_{++}^{n}$ performs huge number of iterations before reaching the superlinear convergence region while the line-search of DNM- $\mathbb{P}_{++}^{n}$ decreasing gradient norm ensuring a superior performance in number of iterations. Note that in these figures DNM- $\mathbb{P}_{++}^{n}$ and NM- $\mathbb{P}_{++}^{n}$ have the same behavior when the iterations close to the solution, which is the consequence of Lemma 4. In part the efficiency of the DNM- $\mathbb{P}_{++}^{n}$ can be explained due to the linearsearch decreasing the merit function.

Table 1 shows that DNM- $\mathbb{P}_{++}^{n}$ is superior to NM- $\mathbb{P}_{++}^{n}$ in number of iterations and $\mathrm{CPU}$ time, besides showing the number of evaluation of the function in the linear-search to compute the stepsize. In the columns of this table we read: $n$ is the dimension of $\mathbb{P}^{n}, b_{1} / a_{1}$ and $b_{2} / a_{2}$ are the parameters defining the functions $f_{1}$ and $f_{2}$, respectively, NIT is the number of iterates to reach the minimaizer 


\begin{tabular}{|c|c|c|c|c|c|c|c|c|c|c|}
\hline & \multirow[b]{2}{*}{$b_{i} / a_{i}$} & \multirow[b]{2}{*}{$n$} & \multicolumn{4}{|c|}{$\mathbf{N M}-\mathbb{P}_{++}^{n}$} & \multicolumn{4}{|c|}{$\mathbf{D N M}-\mathbb{P}_{++}^{n}$} \\
\hline & & & NIT & $\mathrm{HE}$ & GE & $\mathrm{T}$ & NIT & $\mathrm{HE}$ & GE & $\mathrm{T}$ \\
\hline \multirow{9}{*}{$f_{1}$} & \multirow{3}{*}{0.1} & 1 & 41 & 41 & 41 & 0.19 & 4 & 4 & 21 & 0.10 \\
\hline & & 100 & 109 & 109 & 109 & 1.90 & 6 & 6 & 30 & 0.59 \\
\hline & & 1000 & 110 & 110 & 110 & 1240 & 6 & 6 & 30 & 107 \\
\hline & \multirow{3}{*}{1.0} & 1 & 7 & 7 & 7 & 0.10 & 4 & 4 & 17 & 0.11 \\
\hline & & 100 & 13 & 13 & 13 & 0.44 & 4 & 4 & 18 & 0.43 \\
\hline & & 1000 & 13 & 13 & 13 & 147 & 4 & 4 & 18 & 64.90 \\
\hline & \multirow{3}{*}{1.5} & 1 & 6 & 6 & 6 & 0.10 & 4 & 4 & 17 & 0.12 \\
\hline & & 100 & 10 & 10 & 10 & 0.22 & 5 & 5 & 22 & 0.25 \\
\hline & & 1000 & 10 & 10 & 10 & 113 & 6 & 6 & 27 & 97.10 \\
\hline \multirow{9}{*}{$f_{2}$} & \multirow{3}{*}{0.001} & 1 & 249 & 249 & 249 & 0.37 & 6 & 6 & 31 & 0.17 \\
\hline & & 100 & 100 & 100 & 100 & 1.66 & 7 & 7 & 34 & 0.42 \\
\hline & & 1000 & 100 & 100 & 100 & 1130.00 & 7 & 7 & 34 & 131.00 \\
\hline & \multirow{3}{*}{0.002} & 1 & 125 & 125 & 125 & 0.29 & 6 & 6 & 30 & 0.10 \\
\hline & & 100 & 51 & 51 & 51 & 0.90 & 6 & 6 & 29 & 0.27 \\
\hline & & 1000 & 51 & 51 & 51 & 627 & 6 & 6 & 29 & 109 \\
\hline & \multirow{3}{*}{0.01} & 1 & 26 & 26 & 26 & 0.31 & 5 & 5 & 23 & 0.16 \\
\hline & & 100 & 12 & 12 & 12 & 0.92 & 5 & 5 & 22 & 0.21 \\
\hline & & 1000 & 12 & 12 & 12 & 138 & 5 & 5 & 22 & 85.00 \\
\hline
\end{tabular}

Table 1: Performance of DNM- $\mathbb{P}_{++}^{n}$ and NM- $\mathbb{P}_{++}^{n}$ to $f_{1}$ and $f_{2}$ functions.

of the function, HE is the number of Hessian evaluation, GE is the number of Gradient evaluation and $\mathrm{T}$ is the CPU time in seconds. It is important to observe GE in DNM- $\mathbb{P}_{++}^{n}$ takes into account the evaluations in the Armijo's rule also. We know that, in general, the linear-search to compute the stepsize is expensive, but the computational effort did not grow significantly with the dimension of of $\mathbb{P}^{n}$ in the DNM- $\mathbb{P}_{++}^{n}$. In each method we can observe that each iteration evaluates the Hessian once. Hence, DNM- $\mathbb{P}_{++}^{n}$ has a superior performance than NM- $\mathbb{P}_{++}^{n}$ because the minimizer is achieved by DNM- $\mathbb{P}_{++}^{n}$ with less iterates than NM- $\mathbb{P}_{++}^{n}$, as can be seen in Table 1. 


\section{Final Remarks}

In this paper, in order to find a singularity of a vector field defined on Riemannian manifold, we presented a damped Newton's method and established its global convergence with quadratic/superlinear convergence rate. Note that the global convergence analysis of the damped Newton's method without assuming nonsingularity of the hessian of the objective function at its critical points was established in the Euclidean context; see [25, Chapter 1, Section 1.3.3]. Since many important problems in the context of a Riemannian manifold become the problem of finding a singularity of a vector field [39, 1, it would be interesting to obtain a similar global analysis for a damped Newton's method to find a singularity of a vector field defined on a Riemannian manifold. Based on our previous experience, we feel that more tools need to be developed to achieve this goal. In order to obtain a better numerical efficiency to the damped Newton's method our next task is to design new methods where the equation (6) is approximately solved, besides using retraction in 9 and more efficient linear seach in (8).

\section{References}

[1] A. Edelman, T. A. Arias, S. T. Smith, The geometry of algorithms with orthogonality constraints, SIAM J. Matrix Anal. Appl. 20 (2) (1999) 303353.

[2] S. T. Smith, Optimization techniques on Riemannian manifolds, in: Hamiltonian and gradient flows, algorithms and control, Vol. 3 of Fields Inst. Commun., Amer. Math. Soc., Providence, RI, 1994, pp. 113-136.

[3] P.-A. Absil, R. Mahony, R. Sepulchre, Optimization algorithms on matrix manifolds, Princeton University Press, Princeton, NJ, 2008.

[4] P.-A. Absil, L. Amodei, G. Meyer, Two Newton methods on the manifold of fixed-rank matrices endowed with Riemannian quotient geometries, Comput. Statist. 29 (3-4) (2014) 569-590. 
[5] W. Huang, K. A. Gallivan, P.-A. Absil, A Broyden class of quasi-Newton methods for Riemannian optimization, SIAM J. Optim. 25 (3) (2015) 16601685.

[6] C. Li, G. López, V. Martín-Márquez, Monotone vector fields and the proximal point algorithm on Hadamard manifolds, J. Lond. Math. Soc. (2) 79 (3) (2009) 663-683.

[7] C. Li, J. Wang, Newton's method for sections on Riemannian manifolds: generalized covariant $\alpha$-theory, J. Complexity 24 (3) (2008) 423-451.

[8] Y. Hu, C. Li, X. Yang, On convergence rates of linearized proximal algorithms for convex composite optimization with applications, SIAM J. Optim. 26 (2) (2016) 1207-1235.

[9] W. Ring, B. Wirth, Optimization methods on Riemannian manifolds and their application to shape space, SIAM J. Optim. 22 (2) (2012) 596-627.

[10] J. H. Manton, A framework for generalising the Newton method and other iterative methods from Euclidean space to manifolds, Numer. Math. 129 (1) (2015) 91-125.

[11] S. Hosseini, A. Uschmajew, A Riemannian gradient sampling algorithm for nonsmooth optimization on manifolds, SIAM J. Optim. 27 (1) (2017) $173-189$.

[12] P. Grohs, S. Hosseini, Nonsmooth trust region algorithms for locally Lipschitz functions on Riemannian manifolds, IMA J. Numer. Anal. 36 (3) (2016) 1167-1192.

[13] Z. Zhao, Z.-J. Bai, X.-Q. Jin, A Riemannian Newton algorithm for nonlinear eigenvalue problems, SIAM J. Matrix Anal. Appl. 36 (2) (2015) 752774.

[14] L. Cambier, P.-A. Absil, Robust low-rank matrix completion by Riemannian optimization, SIAM J. Sci. Comput. 38 (5) (2016) S440-S460. 
[15] H. Sato, T. Iwai, A Riemannian optimization approach to the matrix singular value decomposition, SIAM J. Optim. 23 (1) (2013) 188-212.

[16] J. E. Dennis, Jr., R. B. Schnabel, Numerical methods for unconstrained optimization and nonlinear equations, Vol. 16 of Classics in Applied Mathematics, Society for Industrial and Applied Mathematics (SIAM), Philadelphia, PA, 1996, corrected reprint of the 1983 original.

[17] J. M. Ortega, W. C. Rheinboldt, Iterative solution of nonlinear equations in several variables, Vol. 30 of Classics in Applied Mathematics, Society for Industrial and Applied Mathematics (SIAM), Philadelphia, PA, 2000, reprint of the 1970 original.

[18] C. Li, J.-H. Wang, J.-P. Dedieu, Smale's point estimate theory for Newton's method on Lie groups, J. Complexity 25 (2) (2009) 128-151.

[19] V. H. Schulz, A Riemannian view on shape optimization, Found. Comput. Math. 14 (3) (2014) 483-501.

[20] J.-H. Wang, C. Li, Kantorovich's theorems for Newton's method for mappings and optimization problems on Lie groups, IMA J. Numer. Anal. 31 (1) (2011) 322-347.

[21] O. P. Ferreira, R. C. M. Silva, Local convergence of Newton's method under a majorant condition in Riemannian manifolds, IMA J. Numer. Anal. 32 (4) (2012) 1696-1713.

[22] O. P. Ferreira, B. F. Svaiter, Kantorovich's theorem on Newton's method in Riemannian manifolds, J. Complexity 18 (1) (2002) 304-329.

[23] C. Li, J. Wang, Newton's method on Riemannian manifolds: Smale's point estimate theory under the $\gamma$-condition, IMA J. Numer. Anal. 26 (2) (2006) 228-251.

[24] T. A. Fernandes, O. P. Ferreira, J. Yuan, On the Superlinear Convergence of Newton's Method on Riemannian Manifolds, J. Optim. Theory Appl. 173 (3) (2017) 828-843. 
[25] D. P. Bertsekas, Constrained optimization and Lagrange multiplier methods, Computer Science and Applied Mathematics, Academic Press, Inc. [Harcourt Brace Jovanovich, Publishers], New York-London, 2014.

[26] A. F. Izmailov, M. V. Solodov, Newton-type methods for optimization and variational problems, Springer Series in Operations Research and Financial Engineering, Springer, Cham, 2014.

[27] O. Burdakov, Some globally convergent modifications of newton's method for solving systems of nonlinear equations, in: Soviet mathematics-Doklady, Vol. 22, 1980, pp. 376-378.

[28] P. Deuflhard, Newton methods for nonlinear problems, Vol. 35 of Springer Series in Computational Mathematics, Springer, Heidelberg, 2011, affine invariance and adaptive algorithms, First softcover printing of the 2006 corrected printing.

[29] P.-A. Absil, C. G. Baker, K. A. Gallivan, Trust-region methods on Riemannian manifolds, Found. Comput. Math. 7 (3) (2007) 303-330.

[30] F. Facchinei, C. Kanzow, A nonsmooth inexact Newton method for the solution of large-scale nonlinear complementarity problems, Math. Programming 76 (3, Ser. B) (1997) 493-512.

[31] M. P. do Carmo, Riemannian geometry, Mathematics: Theory \& Applications, Birkhäuser Boston, Inc., Boston, MA, 1992, translated from the second Portuguese edition by Francis Flaherty.

[32] T. Sakai, Riemannian geometry, Vol. 149 of Translations of Mathematical Monographs, American Mathematical Society, Providence, RI, 1996, translated from the 1992 Japanese original by the author.

[33] J.-P. Dedieu, P. Priouret, G. Malajovich, Newton's method on Riemannian manifolds: convariant alpha theory, IMA J. Numer. Anal. 23 (3) (2003) 395-419. 
[34] O. S. Rothaus, Domains of positivity, Abh. Math. Sem. Univ. Hamburg 24 (1960) 189-235.

[35] U. Helmke, S. Ricardo, S. Yoshizawa, Newton's algorithm in Euclidean Jordan algebras, with applications to robotics, Commun. Inf. Syst. 2 (3) (2002) 283-297, dedicated to the 60th birthday of John B. Moore, Part I.

[36] G. Dirr, U. Helmke, C. Lageman, Nonsmooth Riemannian optimization with applications to sphere packing and grasping, in: Lagrangian and Hamiltonian methods for nonlinear control 2006, Vol. 366 of Lect. Notes Control Inf. Sci., Springer, Berlin, 2007, pp. 29-45.

[37] U. Helmke, K. Hper, J. B. Moore, Quadratically convergent algorithms for optimal dextrous hand grasping, IEEE Transactions on Robotics and Automation 18 (2) (2002) 138-146.

[38] B. N. Datta, Numerical methods for linear control systems: design and analysis, Vol. 1, Academic Press, 2004.

[39] R. L. Adler, J.-P. Dedieu, J. Y. Margulies, M. Martens, M. Shub, Newton's method on Riemannian manifolds and a geometric model for the human spine, IMA J. Numer. Anal. 22 (3) (2002) 359-390. 